\title{
The anomalous current multiplet in 6D minimal supersymmetry
}

\author{
Sergei M. Kuzenko, ${ }^{a}{\text { Joseph } \text { Novak }^{b} \text { and Igor B. Samsonov }}^{a, 1}$ \\ ${ }^{a}$ School of Physics M013, The University of Western Australia, \\ 35 Stirling Highway, Crawley, W.A. 6009, Australia \\ ${ }^{b}$ Max-Planck-Institut für Gravitationsphysik, Albert-Einstein-Institut, \\ Am Mühlenberg 1, D-14476 Golm, Germany \\ E-mail: sergei.kuzenko@uwa.edu.au, joseph.novak@aei.mpg.de, \\ igor.samsonov@uwa.edu.au
}

ABSTRACT: For supersymmetric gauge theories with eight supercharges in four, five and six dimensions, a conserved current belongs to the linear multiplet. In the case of sixdimensional $\mathcal{N}=(1,0)$ Poincaré supersymmetry, we present a consistent deformation of the linear multiplet which describes chiral anomalies. This is achieved by developing a superform formulation for the deformed linear multiplet. In the abelian case, we compute a nonlocal effective action generating the gauge anomaly.

Keywords: Superspaces, Supersymmetric Effective Theories, Anomalies in Field and String Theories

ARXIV EPRINT: 1511.06582

\footnotetext{
${ }^{1}$ On leave from Tomsk Polytechnic University, 634050 Tomsk, Russia.
} 


\section{Contents}

1 Introduction 1

2 Superforms and the anomalous current multiplet $\quad 3$

2.1 The vector multiplet 3

2.2 The superform formulation for the linear multiplet 5

2.3 Chern-Simons couplings to the linear multiplet 6

3 The anomalous effective action $\quad 8$

3.1 Conventional superspace formulation 8

$\begin{array}{lll}3.2 & \text { Harmonic superspace formulation } & 10\end{array}$

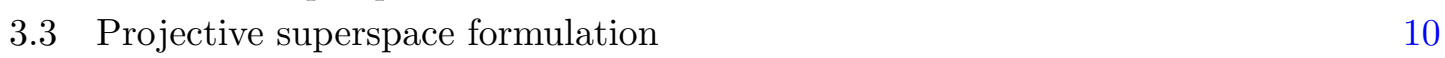

4 An alternative description of the anomalous current multiplet 11

5 Concluding comments 13

A Prepotentials for the Yang-Mills multiplet $\quad 14$

$\begin{array}{ll}\text { A.1 Analytic prepotential } & 14\end{array}$

$\begin{array}{ll}\text { A.2 Mezincescu's prepotential } & 16\end{array}$

$\begin{array}{lll}\text { A.3 Tropical prepotential } & 17\end{array}$

$\begin{array}{ll}\text { B Derivation of the anomalous effective action } & 19\end{array}$

\section{Introduction}

For supersymmetric gauge theories with eight supercharges in four, five and six dimensions, a conserved current belongs to the so-called linear multiplet [1]. This multiplet is described by a real $\mathrm{SU}(2)$ triplet superfield, $L^{i j}=L^{(i j)}$ and $\overline{L^{i j}}=L_{i j}:=\varepsilon_{i k} \varepsilon_{j l} L^{k l}$, subject to the constraint

$$
D_{\alpha}^{(i} L^{j k)}=0
$$

with $\alpha$ the four-component spinor index. Conserved current multiplets in $\mathcal{N}=3$ and $\mathcal{N}=4$ supersymmetric field theories in three dimensions have similar structure $[2,3]$.

It is well known that the four-dimensional $\mathcal{N}=2$ supersymmetric Yang-Mills theories are non-chiral. There are no chiral fermions in five dimensions. Thus all supersymmetric gauge theories with eight supercharges in four and five dimensions are anomaly-free. However, in six dimensions all irreducible matter representations of $\mathcal{N}=(1,0)$ supersymmetry (the hypermultiplet, the vector multiplet and the tensor multiplet) as well as the supergravity multiplet contain chiral fermions. Moreover, the tensor and supergravity multiplets also 
contain chiral bosonic fields, which are gauge two-forms with (anti) self-dual field strengths. These features imply the existence of numerous chiral $\mathcal{N}=(1,0)$ supersymmetric gauge theories. That is why the classification and structure of anomaly-free $6 \mathrm{D}$ supersymmetric theories were thoroughly studied in the 1980s at the component level, see [4] for a review.

In the presence of anomalies, the $6 \mathrm{D}$ conservation equation (1.1) turns into

$$
D_{\alpha}^{(i} L^{j k)}=A_{\alpha}^{i j k},
$$

for some superfield $A_{\alpha}^{i j k}=A_{\alpha}^{(i j k)}$ constrained by ${ }^{1}$

$$
D_{(\alpha}^{(i} A_{\beta)}^{j k l)}=0 .
$$

It was conjectured in [6] that $A_{\alpha}^{i j k}$ has the following structure:

$$
A_{\alpha}^{i j k}=D_{\alpha l} A^{i j k l}, \quad D_{\alpha}^{(i} A^{j k l m)}=0 .
$$

This led the authors of [6] to conclude that the anomalous current multiplet is a $6 \mathrm{D}$ relaxed hypermultiplet [7]. A decade later, this conclusion was re-considered by Howe and Sezgin [8] who studied the $\mathcal{N}=(1,0)$ Yang-Mills multiplet coupled to a tensor multiplet. By analysing the one-loop corrected equations of motion, they found an expression for $A_{\alpha}^{i j k}$ that was incompatible with (1.3).

In this paper we will argue that the anomalous $6 \mathrm{D} \mathcal{N}=(1,0)$ current multiplet $L^{i j}$ obeys the equation

$$
D_{\alpha}^{(i} L^{j k)}=\kappa \mathrm{i} \varepsilon_{\alpha \beta \gamma \delta} \mathcal{W}^{i \beta} \mathcal{W}^{j \gamma} \mathcal{W}^{k \delta}
$$

where $\mathcal{W}^{i \alpha}$ is the field strength of an abelian vector multiplet $[9,10]$, and $\kappa$ a real parameter. As we will show, it follows from this equation that there exists a current $j^{a}$ at the component level satisfying $\partial_{a} j^{a} \propto \varepsilon^{a b c d e f} f_{a b} f_{c d} f_{e f}$, with $f_{a b}$ the component gauge invariant field strength. Equation (1.4) can be shown to be superconformal assuming $L^{i j}$ is a primary operator of dimension 4. On the contrary, it may be shown that the "relaxed hypermultiplet" (1.3) does not describe a representation of the superconformal group.

Equation (1.4) appeared in [8] in the context of the model describing the $6 \mathrm{D} \mathcal{N}=$ $(1,0)$ Yang-Mills multiplet coupled to the tensor multiplet. ${ }^{2}$ Here we will argue that the constraint has a universal nature. ${ }^{3}$ The simplest anomalous $\mathcal{N}=(1,0)$ supersymmetric theory is a hypermultiplet coupled to a $\mathrm{U}(1)$ vector multiplet. The hypermultiplet contains a single chiral fermion interacting with the gauge field. The corresponding gauge anomaly is well known [11-15]

$$
\partial^{a} j_{a}=-\frac{1}{384 \pi^{3}} \varepsilon^{a b c d e f} f_{a b} f_{c d} f_{e f} .
$$

\footnotetext{
${ }^{1}$ The superfield $A_{\alpha}^{i j k}$ subject to the constraint (1.2b) corresponds to a closed six-form, see [5].

${ }^{2}$ The analysis in $[8]$ is rather inconclusive, as may be deduced from the final comments given in that paper. The authors state that "in order to capture the full supermultiplet structure of the equations of motion plus anomalies, one should relax the "relaxed hypermultiplet" even further."

${ }^{3}$ In [8] Howe and Sezgin considered the non-abelian vector multiplet. Eq. (1.4) admits a straightforward generalisation and we leave discussion of it to section 2 .
} 
It corresponds to eq. (1.4) with $\kappa=\frac{1}{96 \pi^{3}}$ and $j_{a}$ a component field of $L^{i j}$ (defined by eq. (2.39)).

This paper is organised as follows. Section 2 gives a superform formulation for the anomalous current multiplet. In particular, the consistency of the constraint (1.4) is demonstrated. In section 3 we compute the nonlocal effective action generating the chiral anomaly. We also discuss how the problem of chiral anomalies should be addressed in the frameworks of the harmonic and the projective superspace approaches. Section 4 is devoted to an alternative description of the anomalous current multiplet. Concluding comments are given in section 5. The paper also includes two technical appendices. Appendix A is devoted to a brief review of the three prepotential formulations for the $6 \mathrm{D} \mathcal{N}=(1,0)$ vector multiplet. Appendix $\mathrm{B}$ contains the technical details concerning the derivation of the anomalous effective action.

\section{Superforms and the anomalous current multiplet}

In this section we present a superform construction for the anomalous current. We work in standard $6 \mathrm{D} \mathcal{N}=(1,0)$ Minkowski superspace parametrised by coordinates $z^{A}=\left(x^{a}, \theta_{i}^{\alpha}\right)$. Our 6D notation and conventions correspond to [16]. In particular, the flat-superspace covariant derivatives are denoted by $D_{A}=\left(\partial_{a}, D_{\alpha}^{i}\right)$, and the dual basis of one-forms is denoted by $E^{A}$, such that $\mathrm{d}=\mathrm{d} z^{M} \partial_{M}=E^{A} D_{A}$.

\subsection{The vector multiplet}

In this subsection we review the superspace formulation for the $6 \mathrm{D} \mathcal{N}=(1,0)$ Yang-Mills supermultiplet following [7]. To describe a non-abelian vector multiplet, the covariant derivative $D_{A}$ has to be replaced with a gauge covariant one,

$$
\mathcal{D}_{A}:=D_{A}+\mathrm{i} \mathcal{V}_{A}
$$

Here the gauge connection one-form $\mathcal{V}=E^{A} \mathcal{V}_{A}$ takes its values in the Lie algebra of the Yang-Mills gauge group $G_{\mathrm{YM}}$. The covariant derivative algebra is

$$
\left[\mathcal{D}_{A}, \mathcal{D}_{B}\right\}=T_{A B}{ }^{C} \mathcal{D}_{C}+\mathrm{i} \mathcal{F}_{A B},
$$

where the only non-vanishing torsion is

$$
T_{\alpha \beta}^{i j c}=-2 \mathrm{i} \varepsilon^{i j}\left(\gamma^{c}\right)_{\alpha \beta}
$$

and $\mathcal{F}_{A B}$ corresponds to the gauge covariant field strength two-form. The covariant derivatives and field strength may be written in a coordinate-free way as follows

$$
\mathcal{D}=\mathrm{d}+\mathrm{i} \mathcal{V}, \quad \mathcal{F}=\mathrm{d} \mathcal{V}-\mathrm{i} \mathcal{V} \wedge \mathcal{V},
$$

where

$$
\mathcal{D}:=\mathrm{d} z^{A} \mathcal{D}_{A}, \quad \mathcal{V}:=\mathrm{d} z^{A} \mathcal{V}_{A}, \quad \mathcal{F}:=\frac{1}{2} \mathrm{~d} z^{B} \wedge \mathrm{d} z^{A} \mathcal{F}_{A B}
$$


The field strength $\mathcal{F}_{A B}$ satisfies the Bianchi identity

$$
\mathcal{D} \mathcal{F}=\mathrm{d} \mathcal{F}+\mathrm{i} \mathcal{V} \wedge \mathcal{F}-\mathrm{i} \mathcal{F} \wedge \mathcal{V}=0 \quad \Longleftrightarrow \quad \mathcal{D}_{[A} \mathcal{F}_{B C\}}-T_{[A B}{ }^{D} \mathcal{F}_{|D| C\}}=0 .
$$

The Yang-Mills gauge transformation acts on the gauge covariant derivatives $\mathcal{D}_{A}$ and a matter superfield $U$ (transforming in some representation of the gauge group) as

$$
\mathcal{D}_{A} \rightarrow \mathrm{e}^{\mathrm{i} \tau} \mathcal{D}_{A} \mathrm{e}^{-\mathrm{i} \tau}, \quad U \rightarrow U^{\prime}=\mathrm{e}^{\mathrm{i} \tau} U, \quad \tau^{\dagger}=\tau,
$$

where the Hermitian gauge parameter $\tau(z)$ takes its values in the Lie algebra of $G_{\mathrm{YM}}$. This implies that the gauge connection and field strength transform as follows

$$
\mathcal{V} \rightarrow \mathrm{e}^{\mathrm{i} \tau} \mathcal{V} \mathrm{e}^{-\mathrm{i} \tau}-\mathrm{ie}^{\mathrm{i} \tau} \mathrm{de}^{-\mathrm{i} \tau}, \quad \mathcal{F} \rightarrow \mathrm{e}^{\mathrm{i} \tau} \mathcal{F} \mathrm{e}^{-\mathrm{i} \tau} .
$$

Some components of the field strength have to be constrained in order to describe an irreducible multiplet. Upon constraining the lowest mass dimension component of the field strength tensor as

$$
\mathcal{F}_{\alpha \beta}^{i j}=0, \quad \mathcal{F}_{a_{\beta}}^{j}=\left(\gamma_{a}\right)_{\beta \gamma} \mathcal{W}^{j \gamma}
$$

the remaining component is completely determined to be

$$
\mathcal{F}_{a b}=-\frac{\mathrm{i}}{8}\left(\gamma_{a b}\right)_{\beta}{ }^{\alpha} \mathcal{D}_{\alpha}^{k} \mathcal{W}_{k}^{\beta}
$$

where the superfield $\mathcal{W}^{i \alpha}$ obeys the Bianchi identities

$$
\mathcal{D}_{\gamma}^{k} \mathcal{W}_{k}^{\gamma}=0, \quad \mathcal{D}_{\alpha}^{(i} \mathcal{W}^{j) \beta}=\frac{1}{4} \delta_{\alpha}^{\beta} \mathcal{D}_{\gamma}^{(i} \mathcal{W}^{j) \gamma} .
$$

The vector indices of $\mathcal{F}_{a b}$ can be converted into spinor ones as follows:

$$
\mathcal{F}_{\alpha}{ }^{\beta}:=-\frac{1}{4}\left(\gamma^{a b}\right)_{\alpha}{ }^{\beta} \mathcal{F}_{a b}=-\frac{\mathrm{i}}{4}\left(\mathcal{D}_{\alpha}^{k} \mathcal{W}_{k}^{\beta}-\frac{1}{4} \delta_{\alpha}^{\beta} \mathcal{D}_{\gamma}^{k} \mathcal{W}_{k}^{\gamma}\right)=-\frac{\mathrm{i}}{4} \mathcal{D}_{\alpha}^{k} \mathcal{W}_{k}^{\beta}
$$

It is convenient to introduce the following superfield:

$$
X^{i j}:=\frac{\mathrm{i}}{4} \mathcal{D}_{\gamma}^{(i} \mathcal{W}^{j) \gamma}
$$

The superfields $\mathcal{W}^{i \alpha}, X^{i j}$ and $\mathcal{F}_{\alpha}{ }^{\beta}$ satisfy the useful identities:

$$
\begin{aligned}
\mathcal{D}_{\alpha}^{i} \mathcal{W}^{j \beta} & =-\mathrm{i} \delta_{\alpha}^{\beta} X^{i j}-2 \mathrm{i} \varepsilon^{i j} \mathcal{F}_{\alpha}{ }^{\beta}, \\
\mathcal{D}_{\alpha}^{i} \mathcal{F}_{\beta}^{\gamma} & =-\mathcal{D}_{\alpha \beta} \mathcal{W}^{i \gamma}-\delta_{\alpha}^{\gamma} \mathcal{D}_{\beta \delta} \mathcal{W}^{i \delta}+\frac{1}{2} \delta_{\beta}^{\gamma} \mathcal{D}_{\alpha \delta} \mathcal{W}^{i \delta}, \\
\mathcal{D}_{\alpha}^{i} X^{j k} & =2 \varepsilon^{i(j} \mathcal{D}_{\alpha \beta} \mathcal{W}^{k) \beta}
\end{aligned}
$$

The above identities indicate how to define the independent component fields contained in $\mathcal{W}^{i \alpha}$. They may be defined as follows:

$$
\lambda^{i \alpha}:=\mathcal{W}^{i \alpha}\left|, \quad f_{\alpha}{ }^{\beta}:=\mathcal{F}_{\alpha}{ }^{\beta}\right|, \quad y^{i j}:=X^{i j} \mid,
$$


where the bar projection of a superfield $U(z)=U(x, \theta)$ is defined by the standard rule $U|:=U(x, \theta)|_{\theta=0}$. The component gauge field is defined by $v_{a}:=\mathcal{V}_{a} \mid$ and is related to the component field strength $f_{a b}$ as follows

$$
f_{a b}=2 \partial_{[a} v_{b]}+\mathrm{i}\left[v_{a}, v_{b}\right] .
$$

It is seen that the vector multiplet consists of the following component fields: $\lambda^{i \alpha}, v_{a}$ and $y^{i j}$.

The supersymmetry transformations of the fields $\lambda^{i \alpha}, v_{a}$ and $y^{i j}$ may be obtained by evaluating the component projection of the identities (2.13). This gives

$$
\begin{aligned}
\delta_{\xi} \lambda^{i \alpha} & =-\mathrm{i} \xi_{j}^{\alpha} y^{i j}+2 \mathrm{i} \xi^{\beta i} f_{\beta}{ }^{\alpha}, \\
\delta_{\xi} y^{i j} & =-2 \xi^{\alpha(i} \mathcal{D}_{\alpha \beta} \lambda^{j) \beta}, \\
\delta_{\xi} v_{a} & =\xi_{j}^{\beta} \mathcal{F}_{\beta}^{j}{ }^{j} \mid=-\xi_{j}^{\alpha}\left(\gamma_{a}\right)_{\alpha \beta} \lambda^{j \beta},
\end{aligned}
$$

where we have used $\mathcal{D}_{a}$ to mean its projection, $\mathcal{D}_{a} \mid=\partial_{a}+\mathrm{i} v_{a}$, when acting on a component field.

\subsection{The superform formulation for the linear multiplet}

The linear multiplet can be described using a four-form gauge potential $B=\frac{1}{4 !} \mathrm{d} z^{D} \wedge \mathrm{d} z^{C} \wedge$ $\mathrm{d} z^{B} \wedge \mathrm{d} z^{A} B_{A B C D}$ possessing the gauge transformation

$$
\delta \mathcal{B}=\mathrm{d} \rho,
$$

where the gauge parameter $\rho$ is an arbitrary three-form. ${ }^{4}$ The corresponding field strength is

$$
H=\mathrm{d} \mathcal{B}=\frac{1}{5 !} \mathrm{d} z^{E} \wedge \mathrm{d} z^{D} \wedge \mathrm{d} z^{C} \wedge \mathrm{d} z^{B} \wedge \mathrm{d} z^{A} H_{A B C D E}
$$

where

$$
H_{A B C D E}=5 D_{[A} \mathcal{B}_{B C D E\}}-10 T_{[A B}^{F} \mathcal{B}_{|F| C D E\}} .
$$

The field strength must satisfy the Bianchi identity

$$
\mathrm{d} H=0 \quad \Longleftrightarrow \quad D_{[A} H_{B C D E F\}}-\frac{5}{2} T_{[A B}{ }^{G} H_{|G| C D E F\}}=0 .
$$

In order to describe the linear multiplet we need to impose some covariant constraints on the field strength $H$. We choose the constraint

$$
H_{a b c_{\alpha \beta}{ }^{i j}}=-2 \mathrm{i}\left(\gamma_{a b c}\right)_{\alpha \beta} L^{i j}, \quad L^{i j}=L^{j i},
$$

and require all lower mass-dimension components to vanish. We can now solve for the remaining components of $H$ in terms of $L^{i j}$. The solution is

$$
\begin{aligned}
H_{a b c d \alpha} i & =-\frac{1}{6} \varepsilon_{a b c d e f}\left(\gamma^{e f}\right)_{\alpha}^{\beta} D_{\beta j} L^{i j}, \\
H_{a b c d e} & =-\frac{\mathrm{i}}{24} \varepsilon_{a b c d e f}\left(\tilde{\gamma}^{f}\right)^{\alpha \beta} D_{\alpha}^{k} D_{\beta}^{l} L_{k l},
\end{aligned}
$$

\footnotetext{
${ }^{4}$ The construction here is a straightforward generalisation of the ones given in [17].
} 
where $L^{i j}$ satisfies the constraint for the linear multiplet

$$
D_{\alpha}^{(i} L^{j k)}=0 .
$$

We defer the definition of the component fields and the explicit form of their supersymmetry transformations to the next subsection.

\subsection{Chern-Simons couplings to the linear multiplet}

Unlike in lower dimensions, the linear multiplet in six dimensions permits a deformation with the use of a non-abelian vector multiplet. The deformed multiplet we will refer to as the deformed linear multiplet. To deform the linear multiplet we now introduce a gauge four-form $\mathcal{B}=\frac{1}{4 !} \mathrm{d} z^{D} \wedge \mathrm{d} z^{C} \wedge \mathrm{d} z^{B} \wedge \mathrm{d} z^{A} \mathcal{B}_{A B C D}$ and its five-form field strength defined by

$$
\mathcal{H}:=\mathrm{d} \mathcal{B}+\kappa \operatorname{tr}\left(\mathcal{V} \wedge \mathcal{F} \wedge \mathcal{F}+\frac{\mathrm{i}}{2} \mathcal{V} \wedge \mathcal{V} \wedge \mathcal{V} \wedge \mathcal{F}-\frac{1}{10} \mathcal{V} \wedge \mathcal{V} \wedge \mathcal{V} \wedge \mathcal{V} \wedge \mathcal{V}\right)
$$

where $\mathcal{V}$ and $\mathcal{F}$ are the Yang-Mills connection and two-form field strength of a non-abelian vector multiplet, respectively. Here $\mathcal{B}$ is understood to be a gauge singlet, $\mathcal{D} \mathcal{B}=\mathrm{d} \mathcal{B}$. The infinitesimal gauge-transformations are

$$
\begin{aligned}
& \delta \mathcal{V}=-\mathrm{d} \tau \\
& \delta \mathcal{B}=\mathrm{d} \rho-\kappa \operatorname{tr}\left(\mathrm{d} \tau \wedge\left(\mathcal{V} \wedge \mathcal{F}+\frac{\mathrm{i}}{2} \mathcal{V} \wedge \mathcal{V} \wedge \mathcal{V}\right)\right),
\end{aligned}
$$

where $\tau$ and $\rho$ generate the gauge transformations of $\mathcal{V}$ and $\mathcal{B}$, respectively. The field strength $\mathcal{H}$ satisfies the Bianchi identity

$$
\mathrm{d} \mathcal{H}=\kappa \operatorname{tr}(\mathcal{F} \wedge \mathcal{F} \wedge \mathcal{F})
$$

which is equivalent to

$$
2 D_{[A} \mathcal{H}_{B C D E F\}}-5 T_{[A B}^{G} \mathcal{H}_{|G| C D E F\}}=30 \kappa \operatorname{tr}\left(\mathcal{F}_{[A B} \mathcal{F}_{C D} \mathcal{F}_{E F\}}\right) .
$$

In order to construct an irreducible multiplet one should constrain the components of $\mathcal{H}$. We can make use of similar constraints as those for the linear multiplet, eq. (2.21). We impose the constraint

$$
\mathcal{H}_{a b c \alpha \beta}^{i j}=-2 \mathrm{i}\left(\gamma_{a b c}\right)_{\alpha \beta} \mathcal{L}^{i j}, \quad \mathcal{L}^{i j}=\mathcal{L}^{j i}
$$

and require all lower components to vanish. Here $\mathcal{L}^{i j}$ is a gauge singlet.

Upon imposing these constraints the remaining components of $\mathcal{H}$ are completely determined and are found to be

$$
\begin{aligned}
\mathcal{H}_{a b c d \alpha}{ }^{i}= & -\frac{1}{6} \varepsilon_{\text {abcdef }}\left(\gamma^{e f}\right)_{\alpha}{ }^{\beta} D_{\beta j} \mathcal{L}^{i j} \\
& +\kappa \mathrm{i} \varepsilon_{a b c d e f}\left(\gamma^{e}\right)_{\alpha \beta}\left(\gamma^{f}\right)_{\gamma \delta} \operatorname{tr}\left(\mathcal{W}_{j}^{\beta} \mathcal{W}^{(j \gamma} \mathcal{W}^{i) \delta}\right), \\
\mathcal{H}_{\text {abcde }}= & \varepsilon_{\text {abcdef }} \tilde{H}^{f},
\end{aligned}
$$


where

$$
D_{\alpha}^{(i} \mathcal{L}^{j k)}=\kappa \mathrm{i} \varepsilon_{\alpha \beta \gamma \delta} \operatorname{tr}\left(\mathcal{W}^{i \beta} \mathcal{W}^{j \gamma} \mathcal{W}^{k \delta}\right)
$$

and

$$
\tilde{\mathcal{H}}^{a}=-\frac{\mathrm{i}}{24}\left(\tilde{\gamma}^{a}\right)^{\alpha \beta} D_{\alpha}^{k} D_{\beta}^{l} \mathcal{L}_{k l}-\frac{\kappa \mathrm{i}}{2} \operatorname{tr}\left(X_{k l}\left(\mathcal{W}^{k} \gamma^{a} \mathcal{W}^{l}\right)\right)+\frac{3 \kappa \mathrm{i}}{8} \operatorname{tr}\left(\mathcal{F}_{b c}\left(\mathcal{W}^{k} \gamma^{a b c} \mathcal{W}_{k}\right)\right)
$$

In deriving the components of $\mathcal{H}$ the following identity proves useful:

$$
\begin{aligned}
D_{\alpha}^{i} \Phi_{\beta}^{j}= & -\frac{1}{2} \varepsilon^{i j} D_{[\alpha}^{k} \Phi_{\beta] k}-\frac{\kappa}{2} \varepsilon_{\alpha \beta \gamma \delta} \operatorname{tr}\left(X_{k}{ }^{i}\left[\mathcal{W}^{j) \gamma}, \mathcal{W}^{k \delta}\right]\right)+\mathrm{i} \partial_{\alpha \beta} \mathcal{L}^{i j} \\
& -\kappa\left(\gamma^{a}\right)_{\alpha \beta}\left(\gamma^{b}\right)_{\gamma \delta} \operatorname{tr}\left(\mathcal{F}_{a b} \mathcal{W}^{(i \gamma} \mathcal{W}^{j) \delta}\right)-\frac{\kappa}{4}\left(\gamma_{a b c}\right)_{\alpha \beta}\left(\gamma^{a}\right)_{\gamma \delta} \operatorname{tr}\left(\mathcal{F}^{b c} \mathcal{W}^{(i \gamma} \mathcal{W}^{j) \delta}\right),
\end{aligned}
$$

where we have defined

$$
\Phi_{\alpha}^{i}=\frac{1}{3} D_{\alpha j} \mathcal{L}^{i j}
$$

It should be noted that the Bianchi identities imply that $\tilde{\mathcal{H}}^{a}$ satisfies

$$
\partial_{a} \tilde{\mathcal{H}}^{a}=\frac{\kappa}{8} \varepsilon^{a b c d e f} \operatorname{tr}\left(\mathcal{F}_{a b} \mathcal{F}_{c d} \mathcal{F}_{e f}\right) .
$$

Using the above results one can deduce the supersymmetry transformations. We define the independent component fields of $\mathcal{L}^{i j}$ as follows:

$$
J^{i j}:=\mathcal{L}^{i j}\left|, \quad \varphi_{\alpha}^{i}:=\Phi_{\alpha}^{i}\right|, \quad b_{a b c d}:=B_{a b c d} \mid .
$$

The component projection of the field strength is related to $b_{a b c d}$ as follows

$$
\mathcal{H}_{a b c d e} \mid=5 \partial_{[a} B_{b c d e]}+\kappa \operatorname{tr}\left(30 v_{[a} f_{b c} f_{d e]}+30 \mathrm{i} v_{[a} v_{b} v_{c} f_{d e]}+12 v_{[a} v_{b} v_{c} v_{d} v_{e]}\right) .
$$

The supersymmetry transformations of the component fields are found with the help of the superform $\mathcal{H}$ and the identity (2.33). They are

$$
\begin{aligned}
\delta_{\xi} J^{i j}= & -2 \xi_{k}^{\alpha} \varphi_{\alpha}^{k}+\kappa \mathrm{i} \xi_{k}^{\beta} \varepsilon_{\beta \gamma \delta \rho} \operatorname{tr}\left(\lambda^{i \gamma} \lambda^{j \delta} \lambda^{k \gamma}\right) \\
\delta_{\xi} \varphi_{\alpha}^{i}= & -\xi^{\beta i}\left(\gamma^{a}\right)_{\beta \alpha} \tilde{\mathcal{H}}_{a} \mid+\kappa \xi^{\beta i} \varepsilon_{\beta \alpha \gamma \delta} \operatorname{tr}\left(y_{k l} \lambda^{k \gamma} \lambda^{l \delta}\right)-3 \kappa \xi^{\beta i} \varepsilon_{\beta \alpha \gamma \rho} \operatorname{tr}\left(f_{\delta}^{\rho}\left[\lambda^{k \gamma}, \lambda_{k}^{\delta}\right]\right) \\
& -\frac{\kappa}{2} \xi_{j}^{\beta} \varepsilon_{\beta \alpha \gamma \delta} \operatorname{tr}\left(y_{k}^{(j}\left[\lambda^{i) \gamma}, \lambda^{k \delta}\right]\right)+\mathrm{i} \xi_{j}^{\beta} \partial_{\beta \alpha} J^{j i}-\kappa \xi_{j}^{\beta}\left(\gamma^{a}\right)_{\beta \alpha}\left(\gamma^{b}\right)_{\gamma \delta} \operatorname{tr}\left(f_{a b} \lambda^{(j \gamma} \lambda^{i) \delta}\right) \\
& -\frac{\kappa}{4} \xi_{j}^{\beta}\left(\gamma_{a b c}\right)_{\beta \alpha}\left(\gamma^{a}\right)_{\gamma \delta} \operatorname{tr}\left(f^{b c} \lambda^{(j \gamma} \lambda^{i) \delta}\right) \\
\delta_{\xi} b_{a b c d}= & -\frac{1}{2} \varepsilon_{a b c d e f} \xi_{k}^{\alpha}\left(\gamma^{e f}\right)_{\alpha}^{\beta} \varphi_{\beta}^{k}+\kappa \mathrm{i} \varepsilon_{a b c d e f} \xi_{j}^{\alpha}\left(\gamma^{e}\right)_{\alpha \beta}\left(\gamma^{f}\right)_{\gamma \delta} \operatorname{tr}\left(\lambda_{k}^{\beta} \lambda^{(j \gamma} \lambda^{k) \delta}\right) \\
& -24 \kappa \operatorname{tr}\left(\delta_{\xi} v_{[a} v_{b} f_{c d]}+\frac{\mathrm{i}}{2} \delta_{\xi} v_{[a} v_{b} v_{c} v_{d]}\right) .
\end{aligned}
$$

The covariant component field strength,

$$
j^{a}:=-2 \tilde{\mathcal{H}}^{a} \mid,
$$


transforms as follows:

$$
\begin{aligned}
\delta_{\xi} j^{a}= & -2\left(\gamma^{a b}\right)_{\alpha}^{\beta} \xi_{i}^{\alpha} \partial_{b} \varphi_{\beta}^{i}+4 \mathrm{i} \kappa \xi_{i}^{\alpha}\left(\gamma^{[a}\right)_{\alpha \beta}\left(\gamma^{b]}\right)_{\gamma \delta} \partial_{b} \operatorname{tr}\left(\lambda_{j}^{\beta} \lambda^{(j \gamma} \lambda^{i) \delta}\right) \\
& +\frac{3 \kappa}{2} \varepsilon^{a b c d e f} \xi_{i}^{\alpha}\left(\gamma_{b}\right)_{\alpha \beta} \operatorname{tr}\left(\lambda^{i \beta} f_{c d} f_{e f}\right) .
\end{aligned}
$$

The supersymmetry transformations for the usual linear multiplet may be obtained by switching off the coupling to the vector multiplet, $\kappa=0$.

The component field $j^{a}$ defined by (2.39) is normalised such that the bar-projection of (2.35) in the abelian case coincides with (1.5) upon identifying $\kappa=1 /\left(96 \pi^{3}\right)$.

\section{The anomalous effective action}

Let $\Gamma$ be an effective action for the abelian vector multiplet. One may think of $\Gamma$ as the functional obtained by integrating out the hypermultiplets coupled to the vector multiplet.

\subsection{Conventional superspace formulation}

If the vector multiplet is described by Mezincescu's prepotential ${ }^{5}[18] M_{i j}(z)$, the effective action is a functional of this superfield,

$$
\Gamma=\Gamma\left[M_{i j}\right] .
$$

Varying $\Gamma$ leads to the functional derivative $L^{i j}=L^{j i}$ defined by

$$
\delta \Gamma=\int \mathrm{d}^{6 \mid 8} z \delta M_{i j} L^{i j},
$$

where the integration is performed over the full superspace. The Mezincescu prepotential $M_{i j}$ has dimension -2 , and its gauge transformation is given by eq. (A.23),

$$
\delta_{\xi} M_{i j}=D_{\alpha}^{k} \xi_{i j k}^{\alpha},
$$

with the gauge parameter $\xi_{i j k}^{\alpha}$ being unconstrained. This gauge transformation means that the theory under consideration is a gauge theory with linearly dependent generators, following the terminology of the Batalin-Vilkovisky quantisation [19]. Indeed, the gauge parameter in (3.3) is defined modulo arbitrary shifts $\xi_{i j k}^{\alpha} \rightarrow \xi_{i j k}^{\alpha}+\delta \xi_{i j k}^{\alpha}$ of the form

$$
\delta \xi_{i j k}^{\alpha}=D_{\beta}^{l} \zeta_{i j k l}^{\alpha \beta}, \quad \zeta_{i j k l}^{\alpha \beta}=\zeta_{(i j k l)}^{(\alpha \beta)}
$$

such that $\delta_{\xi} M_{i j}=\delta_{\xi+\delta \xi} M_{i j}$. In its turn, the parameter $\zeta_{i j k l}^{\alpha \beta}$ in (3.4) is defined modulo arbitrary shifts $\zeta_{i j k l}^{\alpha \beta} \rightarrow \zeta_{i j k l}^{\alpha \beta}+\delta \zeta_{i j k l}^{\alpha \beta}$, where

$$
\delta \zeta_{i j k l}^{\alpha \beta}=D_{\gamma}^{m} \omega_{i j k l m}^{\alpha \beta \gamma}, \quad \omega_{i j k l m}^{\alpha \beta \gamma}=\omega_{(i j k l m)}^{(\alpha \beta \gamma)},
$$

and so forth. This means that the $6 \mathrm{D} \mathcal{N}=(1,0)$ supersymmetric Yang-Mills theory formulated in conventional superspace is a gauge theory of infinite degree of reducibility, similar to the Green-Schwarz superstring.

\footnotetext{
${ }^{5}$ See appendix A for a brief review of the known prepotentials for the vector multiplet.
} 
Under the gauge transformation (3.3) the effective action varies as

$$
\delta_{\xi} \Gamma=\int \mathrm{d}^{6 \mid 8} z \xi_{i j k}^{\alpha} D_{\alpha}^{(k} L^{i j)}
$$

For anomaly-free theories, the effective action is gauge invariant, which means that $L^{i j}$ obeys the conservation equation (1.1). Therefore $L^{i j}$ is a linear multiplet containing a conserved current.

In the presence of anomalies, $L^{i j}$ is no longer a linear multiplet. Instead it obeys the anomalous conservation equation (1.2a). In order for the gauge variation

$$
\delta_{\xi} \Gamma=\int \mathrm{d}^{6 \mid 8} z \xi_{i j k}^{\alpha} A_{\alpha}^{i j k}
$$

to be invariant under the transformation (3.4), the anomaly superfield $A_{\alpha}^{i j k}$ must obey the consistency condition (1.2b). Of course, it must also comply with the Wess-Zumino consistency condition [20]. Both conditions are satisfied if the anomaly superfield is

$$
A_{\alpha}^{i j k}=\mathrm{i} \kappa \varepsilon_{\alpha \beta \gamma \delta} \mathcal{W}^{i \beta} \mathcal{W}^{j \gamma} \mathcal{W}^{k \delta}
$$

for some parameter $\kappa$.

For anomalous theories, the effective action $\Gamma\left[M_{i j}\right]$ may be represented as the sum of two parts,

$$
\Gamma=\Gamma_{A}+\widetilde{\Gamma}
$$

where $\Gamma_{A}$ contains all information about the anomaly, while $\widetilde{\Gamma}$ is a gauge-invariant functional,

$$
\delta_{\xi} \Gamma_{A}=\int \mathrm{d}^{6 \mid 8} z \xi_{i j k}^{\alpha} A_{\alpha}^{i j k}, \quad \delta_{\xi} \widetilde{\Gamma}=0 .
$$

Decomposition (3.9) is not unique. The anomalous part of the effective action, $\Gamma_{A}$, may be determined by making the ansatz

$$
\Gamma_{A}=\int \mathrm{d}^{6 \mid 8} z M_{i j} \Lambda^{i j}
$$

in which $\Lambda^{i j}\left[\mathcal{W}_{k}^{\gamma}\right]$ is a functional of the field strength $\mathcal{W}_{k}^{\gamma}$ subject to the equation

$$
D_{\alpha}^{(k} \Lambda^{i j)}=A_{\alpha}^{i j k}
$$

In appendix B we demonstrate that $\Lambda^{i j}$ may be chosen in the form:

$$
\begin{aligned}
\Lambda^{i j}= & \frac{3 \mathrm{i}}{8} \frac{\partial^{\alpha \beta}}{\square} D_{k \alpha} A_{\beta}^{i j k}+\frac{3}{80} \frac{1}{\square} \varepsilon^{\alpha \beta \gamma \delta} D_{k \alpha} D_{l \beta} D_{\gamma}^{(i} A_{\delta}^{j k l)}-\frac{3}{160} \frac{\partial^{\alpha \beta} \partial^{\mu \nu}}{\square^{2}} D_{k \alpha} D_{l \beta} D_{\mu}^{(i} A_{\nu}^{j k l)} \\
& +\frac{\mathrm{i}}{1152} \frac{\partial_{\alpha \alpha^{\prime}}}{\square^{2}} \varepsilon^{\alpha \beta \gamma \delta} \varepsilon^{\alpha^{\prime} \beta^{\prime} \gamma^{\prime} \delta^{\prime}} D_{k \beta} D_{l \gamma} D_{m \delta} D_{\beta^{\prime}}^{(i} D_{\gamma^{\prime}}^{j} A_{\delta^{\prime}}^{k l m)} \\
& -\frac{1}{64512} \frac{1}{\square^{2}} \varepsilon^{\alpha \beta \gamma \delta} \varepsilon^{\alpha^{\prime} \beta^{\prime} \gamma^{\prime} \delta^{\prime}} D_{k \alpha} D_{l \beta} D_{m \gamma} D_{n \delta} D_{\alpha^{\prime}}^{(i} D_{\beta^{\prime}}^{j} D_{\gamma^{\prime}}^{k} A_{\delta^{\prime}}^{l m n)}
\end{aligned}
$$




\subsection{Harmonic superspace formulation}

In the harmonic superspace approach, the effective action for the vector multiplet is a functional of the analytic prepotential $V^{++}\left(z, u_{i}^{ \pm}\right)$,

$$
\Gamma=\Gamma\left[V^{++}\right] .
$$

Varying $\Gamma$ with respect to $V^{++}$leads to the functional derivative $L^{++}$defined by

$$
\delta \Gamma=\int \mathrm{d} \zeta^{(-4)} \delta V^{++} L^{++}, \quad D_{\alpha}^{+} L^{++}=0 .
$$

In particular, for the gauge variation $\delta_{\lambda} V^{++}=-D^{++} \lambda$, which is the infinitesimal form of (A.13) in the abelian case, we have

$$
\delta_{\lambda} \Gamma=\int \mathrm{d} \zeta^{(-4)} \delta_{\lambda} V^{++} L^{++}=\int \mathrm{d} \zeta^{(-4)} \lambda D^{++} L^{++}
$$

If the theory is anomaly-free, the effective action is gauge invariant, $\delta_{\lambda} \Gamma=0$, and $L^{++}$ obeys the conservation equation

$$
D^{++} L^{++}=0
$$

In the central basis, this equation is equivalent to

$$
L^{++}(z, u)=L^{i j}(z) u_{i}^{+} u_{j}^{+} .
$$

The analyticity condition $D_{\alpha}^{+} L^{++}=0$ means that $L^{i j}$ obeys the conservation equation (1.1). The conserved current multiplet, $L^{i j}$, coincides with the one originating within the conventional superspace formulation described in the previous subsection.

In the presence of anomalies, the conservation equation (3.17) is replaced with

$$
D^{++} L^{++}=A^{(+4)}, \quad D_{\alpha}^{+} A^{(+4)}=0,
$$

with the analytic superfield $A^{(+4)}$ containing all information about the anomaly. The anomaly must obey the Wess-Zumino consistency condition, $\left[\delta_{\lambda_{1}}, \delta_{\lambda_{2}}\right] \Gamma=0$, which is equivalent to

$$
\delta_{\lambda} A^{(+4)}(\zeta)=\int \mathrm{d} \tilde{\zeta}^{(-4)} A^{(4,4)}(\zeta, \tilde{\zeta}) \lambda(\tilde{\zeta}), \quad A^{(4,4)}(\zeta, \tilde{\zeta})=A^{(4,4)}(\tilde{\zeta}, \zeta)
$$

for some bi-analytic kernel $A^{(4,4)}(\zeta, \tilde{\zeta})$.

\subsection{Projective superspace formulation}

In the projective superspace approach, the effective action for the vector multiplet is a functional of the tropical prepotential $V\left(z, v_{i}\right)$,

$$
\Gamma=\Gamma[V] .
$$

Varying $\Gamma$ with respect to $V$ leads to the functional derivative $L^{(2)}(z, v)$, which is a weight-2 projective multiplet, defined by

$$
\delta \Gamma=\frac{1}{2 \pi} \oint_{C}(v, \mathrm{~d} v) \int \mathrm{d}^{6} x D^{(-4)}\left\{\delta V L^{(2)}\right\}, \quad D_{\alpha}^{(1)} L^{(2)}=0,
$$


with $C$ a closed integration contour. Here we have also introduced the fourth-order operator

$$
D^{(-4)}:=-\frac{1}{96} \varepsilon^{\alpha \beta \gamma \delta} D_{\alpha}^{(-1)} D_{\beta}^{(-1)} D_{\gamma}^{(-1)} D_{\delta}^{(-1)}, \quad D_{\alpha}^{(-1)}:=\frac{u_{i}}{(v, u)} D_{\alpha}^{i},
$$

which involves a constant isospinor $u_{i}$ constrained by the only condition $(v, u) \neq 0$ along the integration contour in (3.22). The variation (3.22) may be shown to be invariant under arbitrary projective transformations

$$
\left(u_{i}, v_{i}\right) \rightarrow\left(u_{i}, v_{i}\right) R, \quad R=\left(\begin{array}{cc}
a & 0 \\
b & c
\end{array}\right) \in \mathrm{GL}(2, \mathbb{C}),
$$

and therefore (3.22) is independent of $u_{i}$. It may also be shown that (3.22) is independent of the superspace Grassmann variables.

Choosing $\delta V$ in (3.22) to be an infinitesimal gauge variation (A.30) gives

$$
\delta_{\lambda} \Gamma=\frac{\mathrm{i}}{2 \pi} \oint_{C}(v, \mathrm{~d} v) \int \mathrm{d}^{6} x D^{(-4)}\left\{(\breve{\lambda}-\lambda) L^{(2)}\right\} .
$$

If the theory is anomaly-free, the effective action is gauge invariant, $\delta_{\lambda} \Gamma=0$, for arbitrary weight- 0 arctic superfield $\lambda$. It turns out that this condition implies

$$
L^{(2)}(z, v)=L^{i j}(z) v_{i} v_{j} .
$$

Then the analyticity condition $D_{\alpha}^{(1)} L^{(2)}=0$ means that $L^{i j}$ obeys the constraint (1.1). The conserved current multiplet, $L^{i j}$, coincides with those originating within the conventional and harmonic superspace formulations described in the previous subsections. Eq. (3.26) tells us that associated with the conserved current multiplet $L^{i j}$, eq. (1.1), is the holomorphic tensor field $L^{(2)}$ over $\mathbb{C} P^{1}$.

If the theory is anomalous, the gauge variation (3.25) does not vanish. As a consequence, the projective multiplet $L^{(2)}$ is no longer a linear multiplet.

\section{An alternative description of the anomalous current multiplet}

In section 2 we have constructed the consistent deformation of the $6 \mathrm{D} \mathcal{N}=(1,0)$ linear multiplet given by eq. (2.31). Here an alternative form for the anomalous current multiplet will be derived in the abelian case. We will use some harmonic superspace relations described in subsection A.1.

We associate with the anomalous current multiplet $L^{i j}$, eq. (1.4), the following harmonic superfield:

$$
L^{++}=u_{i}^{+} u_{j}^{+} L^{i j}, \quad D^{++} L^{++}=0 .
$$

Then eq. (1.4) is equivalent to

$$
D_{\alpha}^{+} L^{++}=\kappa \mathrm{i} \varepsilon_{\alpha \beta \gamma \delta} \mathcal{W}^{+\beta} \mathcal{W}^{+\gamma} \mathcal{W}^{+\delta}
$$

with the superfield $\mathcal{W}^{+\alpha}$ being defined by (A.4). 
In the anomaly-free case, the current multiplet $L^{++}=u_{i}^{+} u_{j}^{+} L^{i j}$ is analytic and holomorphic on $\mathbb{C} P^{1}$,

$$
D_{\alpha}^{+} L^{++}=0, \quad D^{++} L^{++}=0 .
$$

Eq. (4.2) tells us that the anomalous current multiplet is no longer analytic.

As a first step, we represent

$$
\mathrm{i} \varepsilon_{\alpha \beta \gamma \delta} \mathcal{W}^{+\beta} \mathcal{W}^{+\gamma} \mathcal{W}^{+\delta}=D_{\alpha}^{+} F^{++},
$$

for some superfield $F^{++}\left(z, u^{ \pm}\right)$defined up to an arbitrary shift of the form

$$
F^{++} \rightarrow F^{++}+H^{++}, \quad D_{\alpha}^{+} H^{++}=0 .
$$

A particular solution of (4.4) is

$$
F^{++}=-\frac{\mathrm{i}}{2} V_{\alpha \beta} \mathcal{W}^{+\alpha} \mathcal{W}^{+\beta}-\frac{\mathrm{i}}{64} \varepsilon^{\alpha \beta \gamma \delta} V_{\alpha \beta} V_{\gamma \delta} D^{+} \mathcal{W}^{+},
$$

where $V_{\alpha \beta}$ is the vector superfield connection defined in (A.14b). In checking (4.4) the following properties of $V_{\alpha \beta}$ may be useful

$$
D_{\alpha}^{+} V_{\beta \gamma}=-2 \varepsilon_{\alpha \beta \gamma \delta} \mathcal{W}^{+\delta}, \quad D^{++} V_{\alpha \beta}=\partial_{\alpha \beta} V^{++} .
$$

It is seen that $F^{++}$is neither analytic nor gauge invariant. However, $D^{++} F^{++}$proves to be analytic,

$$
D^{++} F^{++}=-\frac{\mathrm{i}}{2} G^{++\alpha \beta} \partial_{\alpha \beta} V^{++},
$$

where we have defined

$$
G^{++\alpha \beta}=\mathcal{W}^{+\alpha} \mathcal{W}^{+\beta}+\frac{1}{16} \varepsilon^{\alpha \beta \gamma \delta} V_{\gamma \delta} D^{+} \mathcal{W}^{+}, \quad D_{\gamma}^{+} G^{++\alpha \beta}=0 .
$$

Our second step is to introduce

$$
\mathbb{L}^{++}=L^{++}-\kappa F^{++} .
$$

It follows from (4.2) and (4.4) that $\mathbb{L}^{++}$is analytic,

$$
D_{\alpha}^{+} \mathbb{L}^{++}=0 \text {. }
$$

However, unlike $L^{++}$, the superfield $\mathbb{L}^{++}$is no longer holomorphic on $\mathbb{C} P^{1}$,

$$
D^{++} \mathbb{L}^{++}=\mathbb{A}^{(+4)}, \quad D_{\alpha}^{+} \mathbb{A}^{(+4)}=0 .
$$

The anomaly is now encoded in the analytic superfield $\mathbb{A}^{(+4)}$. It is defined modulo shifts

$$
\mathbb{A}^{(+4)} \rightarrow \mathbb{A}^{(+4)}-\kappa D^{++} H^{++}, \quad D_{\alpha}^{+} H^{++}=0,
$$

where the analytic superfield $H^{++}$is a local composite of the gauge prepotential.

For the choice of $F^{++}$given above, eq. $(4.6), \mathbb{A}^{(+4)}$ is

$$
\mathbb{A}^{(+4)}=\frac{\mathrm{i}}{2} \kappa G^{++\alpha \beta} \partial_{\alpha \beta} V^{++} .
$$

It is an interesting problem to understand whether the functional freedom (4.13) allows one to construct an analytic superfield $A^{(+4)}=\mathbb{A}^{(+4)}-\kappa D^{++} H^{++}$obeying the Wess-Zumino consistency condition (3.20). 


\section{Concluding comments}

In this paper we have presented the consistent deformation, eq. (2.31), of the $6 \mathrm{D} \mathcal{N}=(1,0)$ linear multiplet which describes chiral anomalies. It is

$$
D_{\alpha}^{(i} \mathcal{L}^{j k)}=\kappa \mathrm{i} \varepsilon_{\alpha \beta \gamma \delta} \operatorname{tr}\left(\mathcal{W}^{i \beta} \mathcal{W}^{j \gamma} \mathcal{W}^{k \delta}\right)
$$

Its consistency is guaranteed by the superform formulation for the deformed linear multiplet developed in section 2. Equation (5.1) is superconformal assuming $\mathcal{L}^{i j}$ to be a primary superfield of dimension 4.

The consistent Chern-Simons coupling of the linear multiplet to a vector multiplet, eq. (5.1), is a characteristic feature of $6 \mathrm{D} \mathcal{N}=(1,0)$ supersymmetry. Such a deformation was not possible in the cases of $4 \mathrm{D} \mathcal{N}=2$ and $5 \mathrm{D} \mathcal{N}=1$ supersymmetry. Equation (5.1) is analogous to the constraint describing a deformed $4 \mathrm{D} \mathcal{N}=1$ linear multiplet $\mathcal{L}=\overline{\mathcal{L}}$, which is

$$
\bar{D}^{2} \mathcal{L}=2 \kappa \operatorname{tr}\left(\mathcal{W}^{\alpha} \mathcal{W}_{\alpha}\right), \quad D^{2} \mathcal{L}=2 \kappa \operatorname{tr}\left(\overline{\mathcal{W}}_{\dot{\alpha}} \overline{\mathcal{W}}^{\dot{\alpha}}\right)
$$

with $\mathcal{W}_{\alpha}$ the covariantly chiral field strength of a non-abelian vector multiplet, see [21] for a review of the Chern-Simons couplings to the $4 \mathrm{D} \mathcal{N}=1$ linear multiplet.

In the abelian case, we have computed the nonlocal effective action $\Gamma_{A}$, which is given by the relations (3.11) and (3.13) and which generates the gauge anomaly (3.8). The effective action $\Gamma_{A}$ is constructed as a functional of the Mezincescu prepotential, which corresponds to the formulation of the $6 \mathrm{D} \mathcal{N}=(1,0)$ vector multiplet in conventional superspace $[9,10]$. It is known that such a formulation is not suitable (unlike, e.g., the harmonic superspace approach) to do quantum calculations in general supersymmetric Yang-Mills theories with eight supercharges in diverse dimensions. There are many reasons for that, and the most prominent ones are the following. Firstly, the conventional superspace approach does not offer means to describe off-shell hypermultiplets in complex representations of the gauge group (see [22] for a detailed discussion). Secondly, the Yang-Mills multiplet in this approach is a nontrivial gauge theory with linearly dependent generators of infinite degree of reducibility. As discussed in detail in [23], the BatalinVilkovisky quantisation of the theory has never been used to derive a consistent superfield effective action. ${ }^{6}$ Both problems simply do not occur with the harmonic superspace and the projective superspace approaches.

We computed the effective action (3.11), (3.13) by integrating the gauge anomaly (3.8). However, we did not compute the anomaly by doing supergraph calculations. Once the structure of the anomalous current multiplet is established, it suffices to make use of the known non-supersymmetric results $[11,12,15]$. This is exactly what was done in this paper. It is of interest to compute the gauge anomaly by doing direct supergraph calculations in $6 \mathrm{D} \mathcal{N}=(1,0)$ harmonic superspace, however the existing literature [24-26] does not offer any insight. We hope to report on such calculations elsewhere.

\footnotetext{
${ }^{6}$ Even the Batalin-Vilkovisky quantisation scheme [19] is literally applicable to finitely reducible gauge theories only.
} 


\section{Acknowledgments}

JN is grateful to Daniel Butter for useful discussions. This work is supported in part by the Australian Research Council, project No. DP140103925. JN acknowledges support from GIF - the German-Israeli Foundation for Scientific Research and Development.

\section{A Prepotentials for the Yang-Mills multiplet}

In the case of supersymmetry with eight supercharges in diverse dimensions, $3 \leq d \leq 6$, there exist three different prepotential formulations for the Yang-Mills multiplet, which make use of the following multiplets: (i) the Mezincescu prepotential [18]; (ii) the analytic prepotential [27]; and (iii) the tropical prepotential [28]. The Mezincescu prepotential can be obtained from the analytic one as described in section 7.2.4 of [22]. It can also be read off from the tropical prepotential in accordance with [29]. In its turn, the tropical prepotential can be obtained from the analytic one by getting rid of an infinite tail of superfluous gauge degrees of freedom [30]. In spite of these relationships, the three distinct prepotentials are useful for different applications.

\section{A.1 Analytic prepotential}

Supersymmetric Yang-Mills theory in six-dimensional $\mathcal{N}=(1,0)$ harmonic superspace was formulated in $[31,32]$. Here we briefly review this formulation following the harmonic superspace notation of [22].

Let $u_{i}^{+}$and $u_{i}^{-}$be standard $\mathrm{SU}(2)$ harmonic variables, $\left(u_{i}{ }^{-}, u_{i}{ }^{+}\right) \in \mathrm{SU}(2)$,

$$
\overline{u^{+i}}=u_{i}^{-}, \quad u^{+i} u_{i}^{-}=1,
$$

with $u_{i}^{+}=\varepsilon_{i j} u^{+j}$. Let $D^{++}, D^{--}$and $D^{0}$ be the associated harmonic derivatives defined as in [22]. Using the harmonics we introduce $\mathrm{U}(1)$ projections of the gauge-covariant spinor derivatives

$$
\mathcal{D}_{\alpha}^{ \pm}=u_{i}^{ \pm} \mathcal{D}_{\alpha}^{i}=D_{\alpha}^{ \pm}+\mathrm{i} \mathcal{V}_{\alpha}^{ \pm}, \quad \mathcal{V}_{\alpha}^{ \pm}=u_{i}^{ \pm} \mathcal{V}_{\alpha}^{i}
$$

In accordance with (2.2), the operators (A.2) obey the following (anti)commutation relations

$$
\begin{aligned}
\left\{\mathcal{D}_{\alpha}^{+}, \mathcal{D}_{\beta}^{+}\right\} & =0 \\
\left\{\mathcal{D}_{\alpha}^{+}, \mathcal{D}_{\beta}^{-}\right\} & =2 \mathrm{i}\left(\gamma^{a}\right)_{\alpha \beta} \mathcal{D}_{a}, \\
{\left[\mathcal{D}_{a}, \mathcal{D}_{\alpha}^{ \pm}\right] } & =\mathrm{i}\left(\gamma_{a}\right)_{\alpha \beta} \mathcal{W}^{ \pm \beta}, \\
{\left[\mathcal{D}_{a}, \mathcal{D}_{b}\right] } & =\mathrm{i} \mathcal{F}_{a b},
\end{aligned}
$$

where $\mathcal{W}^{ \pm \alpha}$ are the irreducible $\mathrm{U}(1)$ components of the field strength $\mathcal{W}^{i \alpha}$,

$$
\mathcal{W}^{ \pm \alpha}=u_{i}^{ \pm} \mathcal{W}^{i \alpha}
$$

In the harmonic superspace setting, it is useful to combine the superspace gaugecovariant derivatives with the harmonic ones,

$$
\mathcal{D}_{\hat{A}}=\left(\mathcal{D}_{a}, \mathcal{D}_{\alpha}^{ \pm}, \mathcal{D}^{++}, \mathcal{D}^{--}, \mathcal{D}^{0}\right):=\left(\mathcal{D}_{a}, \mathcal{D}_{\alpha}^{ \pm}, D^{++}, D^{--}, D^{0}\right)=D_{\hat{A}}+\mathrm{i} \mathcal{V}_{\hat{A}}
$$


The gauge transformation of $\mathcal{D}_{\hat{A}}$ is analogous to (2.7),

$$
\mathcal{D}_{\hat{A}} \longrightarrow \mathcal{D}_{\hat{A}}=\mathrm{e}^{\mathrm{i} \tau} \mathcal{D}_{\hat{A}} \mathrm{e}^{-\mathrm{i} \tau} .
$$

Since the gauge superfield parameter $\tau$ is harmonic independent, the harmonic derivatives $\left(D^{ \pm \pm}, D^{0}\right)$ are gauge covariant.

The equation (A.3a) is the integrability condition for covariantly analytic superfields to exist. This equation can be solved in terms of a bridge superfield $b=b(z, u)$ defined by the rule

$$
\mathcal{D}_{\alpha}^{+}=\mathrm{e}^{-\mathrm{i} b} D_{\alpha}^{+} \mathrm{e}^{\mathrm{i} b} .
$$

The introduction of the bridge superfield leads to a new gauge freedom, in addition to the $\tau$-gauge transformations (2.7). The complete gauge transformation law of $b$ is

$$
\mathrm{e}^{\mathrm{i} b^{\prime}}=\mathrm{e}^{\mathrm{i} \lambda} \mathrm{e}^{\mathrm{i} b} \mathrm{e}^{-\mathrm{i} \tau}
$$

where $\lambda$ is a $\mathrm{U}(1)$ neutral analytic superfield, $D_{\alpha}^{+} \lambda=0$.

The representation (A.5) for the gauge-covariant derivatives is called the $\tau$-frame. The bridge superfield allows one to introduce a new representation for the gauge-covariant derivatives, which is defined by

$$
\mathcal{D}_{\hat{A}} \longrightarrow \nabla_{\hat{A}}=\mathrm{e}^{\mathrm{i} b} \mathcal{D}_{\hat{A}} \mathrm{e}^{-\mathrm{i} b}=D_{\hat{A}}+\mathrm{i} V_{\hat{A}}
$$

and is called the $\lambda$-frame. In this frame, the derivative $\nabla_{\alpha}^{+}$is short, $\nabla_{\alpha}^{+}=D_{\alpha}^{+}$, and hence $V_{\alpha}^{+}=0$. However, two of the three harmonic derivatives acquire gauge connections:

$$
\nabla^{++}=D^{++}+\mathrm{i} V^{++}, \quad \nabla^{--}=D^{--}+\mathrm{i} V^{--} .
$$

As follows from the commutation relation $\left[\nabla_{\alpha}^{+}, \nabla^{++}\right]=0$, the gauge connection $V^{++}$ is analytic,

$$
D_{\alpha}^{+} V^{++}=0 .
$$

The connection $V^{--}$can be expressed via $V^{++}$as a unique solution of the zero-curvature condition

$$
\left[\nabla^{++}, \nabla^{--}\right]=D^{0} \quad \Longleftrightarrow \quad D^{++} V^{--}-D^{--} V^{++}+\mathrm{i}\left[V^{++}, V^{--}\right]=0
$$

The explicit expression for $V^{--}$in terms of $V^{++}$was originally found by Zupnik [33]. In the $\lambda$-frame, no $\tau$-gauge freedom remains. Under the $\lambda$-gauge group, the connections $V^{++}$ and $V^{--}$transform as

$$
V^{\prime \pm \pm}=\mathrm{e}^{\mathrm{i} \lambda} V^{ \pm \pm} \mathrm{e}^{-\mathrm{i} \lambda}-\mathrm{i} \mathrm{e}^{\mathrm{i} \lambda} D^{ \pm \pm} \mathrm{e}^{-\mathrm{i} \lambda}
$$

The $\lambda$-frame counterparts of the field strengths $\mathcal{W}^{ \pm \alpha}$ will be denoted $W^{ \pm \alpha}$. In the abelian case, there is no difference between $\mathcal{W}^{ \pm \alpha}$ and $W^{ \pm \alpha}$.

The $\lambda$-frame counterparts of the (anti-)commutation relations (A.3b) and (A.3c), in conjunction with the identity $\left[\nabla^{--}, \nabla_{\alpha}^{+}\right]=\nabla_{\alpha}^{-}$, allow one to express the gauge connections 
$V_{\alpha}^{-}$and $V_{a}$ and the field strength $W^{+\alpha}$ in terms $V^{--}$. The explicit expressions for the connections are

$$
\begin{aligned}
V_{\alpha}^{-} & =-D_{\alpha}^{+} V^{--} \\
V_{a} & =\frac{\mathrm{i}}{8}\left(\tilde{\gamma}_{a}\right)^{\alpha \beta} D_{\alpha}^{+} D_{\beta}^{+} V^{--} \quad \Longleftrightarrow \quad V_{\alpha \beta}=\left(\gamma^{a}\right)_{\alpha \beta} V_{a}=\frac{\mathrm{i}}{2} D_{\alpha}^{+} D_{\beta}^{+} V^{--} .
\end{aligned}
$$

The expression for the field strength is

$$
W^{+\alpha}=\frac{\mathrm{i}}{24} \varepsilon^{\alpha \beta \gamma \delta} D_{\beta}^{+} D_{\gamma}^{+} D_{\delta}^{+} V^{--} .
$$

As mentioned above, $V^{--}$is uniquely expressed in terms of the analytic connection $V^{++}$. Thus, the superfield $V^{++}$is a single prepotential in terms of which all the connections are determined, in complete analogy with the $4 \mathrm{D}$ case [27]. This prepotential is analytic, but otherwise unconstrained.

\section{A.2 Mezincescu's prepotential}

The Mezincescu prepotential was used in $[9,10]$ to describe the $6 \mathrm{D} \mathcal{N}=(1,0)$ vector multiplet in Minkowski superspace. In this subsection we recall how the Mezincescu prepotential is obtained from the analytic one following the discussion in section 7.2.4 of [22]. Only the abelian vector multiplet is considered here.

In the harmonic superspace approach, the gauge prepotential $V^{++}$and the gauge parameter $\lambda$ are analytic superfields, $D_{\alpha}^{+} V^{++}=0$ and $D_{\alpha}^{+} \lambda=0$. The analyticity constraint on $V^{++}$is solved by

$$
V^{++}=\left(D^{+}\right)^{4} M^{--}
$$

where

$$
\left(D^{+}\right)^{4}=-\frac{1}{96} \varepsilon^{\alpha \beta \gamma \delta} D_{\alpha}^{+} D_{\beta}^{+} D_{\gamma}^{+} D_{\delta}^{+}
$$

is the analytic projection operator, and $M^{--}(z, u)$ is an unconstrained superfield. Similarly, the analyticity constraint on $\lambda$ is solved by

$$
\lambda=\left(D^{+}\right)^{4} \rho^{(-4)},
$$

where $\rho^{(-4)}(z, u)$ is an unconstrained superfield. The original $\lambda$-transformation of $V^{++}$ turns into the following gauge transformation of $M^{--}$:

$$
\delta_{\lambda} M^{--}=-D^{++} \rho^{(-4)} .
$$

In addition, it follows from (A.16) that $M^{--}$possesses a new gauge freedom that leaves $V^{++}$invariant and acts on $M^{--}$as follows:

$$
\delta_{\xi} M^{--}=D_{\alpha}^{+} \xi^{(-3) \alpha} .
$$

Here $\xi^{(-3) \alpha}(z, u)$ is an unconstrained gauge parameter.

The superfields $M^{--}(z, u), \rho^{(-4)}(z, u)$ and $\xi^{(-3) \alpha}(z, u)$ are smooth scalar fields on the group manifold $\mathrm{SU}(2)$ of definite $\mathrm{U}(1)$ charges or, equivalently, smooth tensor fields on the 
two-sphere $S^{2}=\mathrm{SU}(2) / \mathrm{U}(1)$. Therefore these superfields are given by convergent Fourier series in the harmonic variables,

$$
\begin{aligned}
M^{--}(z, u) & =M^{i j}(z) u_{i}^{-} u_{j}^{-}+M^{i j k l}(z) u_{(i}^{+} u_{j}^{-} u_{k}^{-} u_{l)}^{-}+\ldots \\
\rho^{(-4)}(z, u) & =\rho^{i j k l}(z) u_{i}^{-} u_{j}^{-} u_{k}^{-} u_{l}^{-}+\ldots \\
\xi^{(-3) \alpha}(z, u) & =\frac{4}{3} \xi^{i j k \alpha}(z) u_{i}^{-} u_{j}^{-} u_{k}^{-}+\ldots
\end{aligned}
$$

where the numerical coefficient in the last relation is introduced for later convenience. Comparing the series (A.21a) and (A.21b), one can see that the gauge freedom (A.19) allows one to gauge away all Fourier components of $M^{--}$in (A.21a) except for the lowest one. In other words, one can impose a supersymmetric gauge

$$
M^{--}(z, u)=M^{i j}(z) u_{i}^{-} u_{j}^{-}, \quad M^{i j}=M^{(i j)} .
$$

The remaining superfield $M^{i j}$ is exactly Mezincescu's prepotential [18].

The gauge condition (A.22) completely fixes the $\rho$-gauge freedom. However, there remains a residual $\xi$-invariance generated solely by the spinor gauge parameter $\xi^{i j k \alpha}=$ $\xi^{(i j k) \alpha}$ in the series (A.21c). It acts on Mezincescu's prepotential by the rule

$$
\delta_{\xi} M_{i j}=D_{\alpha}^{k} \xi_{i j k}^{\alpha},
$$

which is exactly the gauge transformation derived in $[9,10]$. In order to preserve the gauge condition (A.22), this $\xi$-transformation has to be accompanied by a special $\rho$ transformation

$$
\rho^{(-4)}(z, u)=\frac{1}{3} D_{\alpha}^{i} \xi^{j k l \alpha}(z) u_{i}^{-} u_{j}^{-} u_{k}^{-} u_{l}^{-}
$$

\section{A.3 Tropical prepotential}

Here we recall the definition of the tropical prepotential [28] which is used to describe the Yang-Mills multiplet within the projective superspace approach [28, 34]. We follow the modern presentation of this approach given, e.g., in [35].

In the projective superspace setting, one does not work with the harmonics used in subsection A.1. Instead, one deals with homogeneous coordinates $v^{i} \in \mathbb{C}^{2} \backslash\{0\}$ for $\mathbb{C} P^{1}$. We recall that $\mathbb{C} P^{1}$ is obtained from $\mathbb{C}^{2} \backslash\{0\}$ by factorisation with respect to the equivalence relation $v^{i} \sim c v^{i}$, with $c \in \mathbb{C}^{*}$. Supersymmetric field theories are described in terms of the so-called weight- $n$ projective multiplets $Q^{(n)}(z, v)$. By definition, such a superfield is defined by the following conditions:

(i) $Q^{(n)}(z, v)$ is holomorphic over an open domain of $\mathbb{C} P^{1}$,

$$
\frac{\partial}{\partial \bar{v}_{i}} Q^{(n)}=0
$$

(ii) it is a homogeneous function of $v^{i}$ of degree $n$,

$$
Q^{(n)}(z, c v)=c^{n} Q^{(n)}(z, v), \quad c \in \mathbb{C}^{*} .
$$


(iii) it obeys the analyticity condition

$$
D_{\alpha}^{(1)} Q^{(n)}=0, \quad D_{\alpha}^{(1)}=v_{i} D_{\alpha}^{i}
$$

Introduce two special points in $N, S \in \mathbb{C} P^{1}$ : the north pole $N$ with homogeneous coordinates $v^{i} \propto(0,1)$, and the south pole $S$ labeled by $v^{i} \propto(1,0)$. Associated with these points are two open domains, the north chart $\mathbb{C} P^{1} \backslash\{N\}$ and the south chart $\mathbb{C} P^{1} \backslash$ $\{S\}$, which cover $\mathbb{C} P^{1}$. In the north chart, we can introduce a complex (inhomogeneous) coordinate $\zeta$ as

$$
v^{i}=v^{\underline{1}}(1, \zeta), \quad \zeta:=\frac{v^{\underline{2}}}{v^{\underline{1}}}, \quad i=\underline{1}, \underline{2} .
$$

The tropical multiplet $V(z, v)$ is a weight-0 projective multiplet holomorphic on $\mathbb{C} P^{1} \backslash$ $\{N \cup S\}$. It is also constrained to be real under the so-called smile conjugation, see [35] for more details. It is given by a Laurent series

$$
V(z, v)=V(z, \zeta)=\sum_{n=-\infty}^{\infty} V_{n}(z) \zeta^{n}, \quad V_{n}^{\dagger}=(-1)^{n} V_{-n}
$$

The gauge transformation law of the tropical prepotential is

$$
\mathrm{e}^{V^{\prime}}=\mathrm{e}^{\mathrm{i} \breve{\lambda}} \mathrm{e}^{V} \mathrm{e}^{-\mathrm{i} \lambda}
$$

where the gauge parameter $\lambda(z, \zeta)$ is a weight-zero arctic multiplet

$$
D_{\alpha}^{(1)} \lambda=0, \quad \lambda(z, \zeta)=\sum_{n=0}^{\infty} \lambda_{n}(z) \zeta^{n},
$$

and its smile conjugated antarctic multiplet,

$$
\breve{\lambda}(z, \zeta)=\sum_{k=0}^{\infty}(-1)^{k} \lambda_{k}^{\dagger}(z) \frac{1}{\zeta^{k}} .
$$

By definition, a weight- $n$ arctic multiplet is holomorphic on $\mathbb{C} P^{1} \backslash\{N\}$.

Modulo purely gauge degrees of freedom, the gauge-covariant derivatives can be expressed in terms of the tropical prepotential. This is explained in detail in the cases of 3D $\mathcal{N}=4$ and $5 \mathrm{D} \mathcal{N}=1$ vector multiplets coupled to conformal supergravity in [36] and [37] respectively. The $6 \mathrm{D} \mathcal{N}=(1,0)$ case can be treated similarly.

Following [29], the Mezincescu prepotential is introduced by the rule

$$
M_{i j}(z)=\frac{1}{2 \pi} \oint_{C}(v, \mathrm{~d} v) v_{i} v_{j} U^{(-4)}(z, v), \quad(v, \mathrm{~d} v)=v^{k} \mathrm{~d} v_{k},
$$

where $U^{(-4)}(z, v)$ is related to the tropical prepotential as follows:

$$
V(z, v)=D^{(4)} U^{(-4)}(z, v), \quad D^{(4)}=-\frac{1}{96} \varepsilon^{\alpha \beta \gamma \delta} D_{\alpha}^{(1)} D_{\beta}^{(1)} D_{\gamma}^{(1)} D_{\delta}^{(1)} .
$$




\section{B Derivation of the anomalous effective action}

To find the functional generating the anomalous effective action (3.11) it suffices to find a particular solution of the equation (3.12) which we denote by $\Lambda^{i j}$. In this appendix we will demonstrate that a particular solution of this equation can be represented in the form (3.13).

Given the superfields $\Lambda^{i j}$ and $A_{\alpha}^{i j k}$ it is convenient to deal with their harmonic projections

$$
\Lambda^{++}=u_{i}^{+} u_{j}^{+} \Lambda^{i j}, \quad A_{\alpha}^{(+3)}=u_{i}^{+} u_{j}^{+} u_{k}^{+} A_{\alpha}^{i j k} .
$$

Then the equation (3.12) is equivalent to

$$
D_{\alpha}^{+} \Lambda^{++}=A_{\alpha}^{(+3)}
$$

where $D_{\alpha}^{+}=u_{i}^{+} D_{\alpha}^{i}$. Note that, by construction, the superfield $\Lambda^{++}$obeys

$$
D^{++} \Lambda^{++}=0 \text {. }
$$

We also point out that $A_{\alpha}^{(+3)}$ satisfies

$$
D_{(\alpha}^{+} A_{\beta)}^{(+3)}=0 \quad \Longrightarrow \quad\left(D^{+}\right)^{4} A_{\alpha}^{(+3)}=0,
$$

as a consequence of $(1.2 \mathrm{~b})$.

We look for a solution of the equation (B.2) in the form of the sum of the following terms

$$
\Lambda^{++}=\sum_{i=1}^{9} c_{i} \Lambda_{i}^{++},
$$

where $c_{i}$ are some coefficients and

$$
\begin{aligned}
\Lambda_{1}^{++} & =\frac{\mathrm{i}}{2} \frac{\partial^{\alpha \beta}}{\square} D_{\alpha}^{-} A_{\beta}^{+++}, \\
\Lambda_{2}^{++} & =\mathrm{i} \frac{\partial^{\alpha \beta}}{\square} D^{--} D_{\alpha}^{+} A_{\beta}^{+++}, \\
\Lambda_{3}^{++} & =\frac{1}{\square} \varepsilon^{\alpha \beta \gamma \delta} D_{\alpha}^{-} D_{\beta}^{-} D_{\gamma}^{+} A_{\delta}^{+++}, \\
\Lambda_{4}^{++} & =\frac{1}{\square} D^{--} \varepsilon^{\alpha \beta \gamma \delta} D_{\alpha}^{-} D_{\beta}^{+} D_{\gamma}^{+} A_{\delta}^{+++}, \\
\Lambda_{5}^{++} & =\frac{1}{\square} D^{--} D^{--} \varepsilon^{\alpha \beta \gamma \delta} D_{\alpha}^{+} D_{\beta}^{+} D_{\gamma}^{+} A_{\delta}^{+++}, \\
\Lambda_{6}^{++} & =\frac{\partial^{\alpha \beta} \partial^{\mu \nu}}{\square^{2}} D_{\alpha}^{-} D_{\beta}^{-} D_{\mu}^{+} A_{\nu}^{+++}, \\
\Lambda_{7}^{++} & =\mathrm{i} \frac{\partial_{\alpha \alpha^{\prime}}}{\square^{2}} \varepsilon^{\alpha \beta \gamma \delta} D_{\beta}^{-} D_{\gamma}^{-} D_{\delta}^{-} \varepsilon^{\alpha^{\prime} \beta^{\prime} \gamma^{\prime} \delta^{\prime}} D_{\beta^{\prime}}^{+} D_{\gamma^{\prime}}^{+} A_{\delta^{\prime}}^{+++}, \\
\Lambda_{8}^{++} & =\mathrm{i} \frac{\partial^{\mu \nu}}{\square^{2}} D^{--} D_{\mu}^{-} D_{\nu}^{-} \varepsilon^{\alpha \beta \gamma \delta} D_{\alpha}^{+} D_{\beta}^{+} D_{\gamma}^{+} A_{\delta}^{+++}, \\
\Lambda_{9}^{++} & =\frac{1}{\square^{2}} \varepsilon^{\alpha \beta \gamma \delta}\left(D^{-}\right)^{4} D_{\alpha}^{+} D_{\beta}^{+} D_{\gamma}^{+} A_{\delta}^{+++} .
\end{aligned}
$$


The equation (B.3) is satisfied on the condition that

$$
\begin{aligned}
c_{1}+8 c_{2}+8 c_{3}+16 c_{6} & =0, \\
c_{3}+2 c_{4}+\frac{1}{3} c_{6}-6 c_{7} & =0, \\
c_{4}+10 c_{5}-8 c_{8} & =0, \\
3 c_{7}+8 c_{8}-\frac{1}{2} c_{9} & =0 .
\end{aligned}
$$

Imposing the equation (B.2) we find the following constraints for the coefficients $c_{i}$ :

$$
\begin{aligned}
c_{1} & =1, \\
c_{1}+4 c_{2}+16 c_{6} & =0, \\
c_{1}-16 c_{3} & =0, \\
c_{2}-6 c_{4} & =0, \\
\frac{2}{3} c_{3}+c_{4}-12 c_{7} & =0, \\
c_{4}+8 c_{5}-16 c_{8} & =0, \\
c_{6}+18 c_{7} & =0, \\
4 c_{8}+3 c_{7}-c_{9} & =0 .
\end{aligned}
$$

The solution of the system of equations (B.7) and (B.8) reads

$$
\begin{aligned}
& c_{1}=1, \quad c_{2}=-\frac{1}{8}, \quad c_{3}=\frac{1}{16}, \quad c_{4}=-\frac{1}{48}, \quad c_{5}=\frac{1}{576}, \\
& c_{6}=-\frac{1}{32}, \quad c_{7}=\frac{1}{576}, \quad c_{8}=-\frac{1}{2304}, \quad c_{9}=\frac{1}{288} .
\end{aligned}
$$

Note that different terms in (B.5) depend on different harmonic monomials. Nevertheless, the equation (B.3) guarantees that the full expression (B.5) is quadratic in harmonics in agreement with (B.1). Therefore, we can restore $\Lambda^{i j}$ from $\Lambda^{++}$by the rule

$$
\Lambda^{i j}=3 \int \mathrm{d} u u^{-i} u^{-j} \Lambda^{++}
$$

The harmonic integral is computed according to the formula [38]

$$
\int \mathrm{d} u u^{+i_{1}} \ldots u^{+i_{n}} u_{j_{1}}^{-} \ldots u_{j_{n}}^{-}=\frac{1}{n+1} \delta_{\left(j_{1}\right.}^{i_{1}} \ldots \delta_{\left.j_{n}\right)}^{i_{n}} .
$$

Applying this rule to all terms in the sum (B.5) we find

$$
\begin{aligned}
\Lambda^{i j}= & 3 \sum_{k=1}^{9} c_{k} \int \mathrm{d} u u^{-i} u^{-j} \Lambda_{k}^{++} \\
= & \frac{3 \mathrm{i}}{8} \frac{\partial^{\alpha \beta}}{\square} D_{k \alpha} A_{\beta}^{i j k}+\frac{3}{80} \frac{1}{\square} \varepsilon^{\alpha \beta \gamma \delta} D_{k \alpha} D_{l \beta} D_{\gamma}^{(i} A_{\delta}^{j k l)}-\frac{3}{160} \frac{\partial^{\alpha \beta} \partial^{\mu \nu}}{\square^{2}} D_{k \alpha} D_{l \beta} D_{\mu}^{(i} A_{\nu}^{j k l)} \\
& +\frac{\mathrm{i}}{1152} \frac{\partial_{\alpha \alpha^{\prime}}}{\square^{2}} \varepsilon^{\alpha \beta \gamma \delta} \varepsilon^{\alpha^{\prime} \beta^{\prime} \gamma^{\prime} \delta^{\prime}} D_{k \beta} D_{l \gamma} D_{m \delta} D_{\beta^{\prime}}^{(i} D_{\gamma^{\prime}}^{j} A_{\delta^{\prime}}^{k l m)} \\
& \left.-\frac{1}{64512} \frac{1}{\square^{2}} \varepsilon^{\alpha \beta \gamma \delta} \varepsilon^{\alpha^{\prime} \beta^{\prime} \gamma^{\prime} \delta^{\prime}} D_{k \alpha} D_{l \beta} D_{m \gamma} D_{n \delta} D_{\alpha^{\prime}}^{(i} D_{\beta^{\prime}}^{j} D_{\gamma^{\prime}}^{k} A_{\delta^{\prime}}^{l m n}\right)
\end{aligned}
$$

Note that the terms $\Lambda_{2}^{++}, \Lambda_{4}^{++}, \Lambda_{5}^{++}$and $\Lambda_{8}^{++}$do not contribute to this expression owing to the identity [27] $\int \mathrm{d} u D^{--} F^{++}=0$, for any smooth field $F^{++}(u)$. 
Open Access. This article is distributed under the terms of the Creative Commons Attribution License (CC-BY 4.0), which permits any use, distribution and reproduction in any medium, provided the original author(s) and source are credited.

\section{References}

[1] P. Breitenlohner and M.F. Sohnius, Superfields, auxiliary fields and tensor calculus for $N=2$ extended supergravity, Nucl. Phys. B 165 (1980) 483 [InSPIRE].

[2] E.I. Buchbinder, S.M. Kuzenko and I.B. Samsonov, Superconformal field theory in three dimensions: correlation functions of conserved currents, JHEP 06 (2015) 138 [arXiv: 1503.04961] [INSPIRE].

[3] E.I. Buchbinder, S.M. Kuzenko and I.B. Samsonov, Implications of $\mathcal{N}=4$ superconformal symmetry in three spacetime dimensions, JHEP 08 (2015) 125 [arXiv:1507.00221] [INSPIRE].

[4] A. Salam and E. Sezgin, Supergravities in diverse dimensions, volume 2, World Scientific, Singapore (1989).

[5] C. Arias, W.D. Linch, III and A.K. Ridgway, Superforms in simple six-dimensional superspace, arXiv: 1402.4823 [INSPIRE].

[6] P.S. Howe and A. Umerski, Anomaly multiplets in six-dimensions and ten-dimensions, Phys. Lett. B 198 (1987) 57 [INSPIRE].

[7] P.S. Howe, K.S. Stelle and P.K. Townsend, The relaxed hypermultiplet: an unconstrained $N=2$ superfield theory, Nucl. Phys. B 214 (1983) 519 [InSPIRE].

[8] P.S. Howe and E. Sezgin, Anomaly free tensor Yang-Mills system and its dual formulation, Phys. Lett. B 440 (1998) 50 [hep-th/9806050] [InSPIRE].

[9] P.S. Howe, G. Sierra and P.K. Townsend, Supersymmetry in six-dimensions, Nucl. Phys. B 221 (1983) 331 [INSPIRE].

[10] J. Koller, A six-dimensional superspace approach to extended superfields, Nucl. Phys. B 222 (1983) 319 [INSPIRE].

[11] P.H. Frampton and T.W. Kephart, Explicit evaluation of anomalies in higher dimensions, Phys. Rev. Lett. 50 (1983) 1343 [Erratum ibid. 51 (1983) 232] [INSPIRE].

[12] P.H. Frampton and T.W. Kephart, The analysis of anomalies in higher space-time dimensions, Phys. Rev. D 28 (1983) 1010 [InSPIRE].

[13] L. Álvarez-Gaumé and E. Witten, Gravitational anomalies, Nucl. Phys. B 234 (1984) 269 [INSPIRE].

[14] B. Zumino, Y.-S. Wu and A. Zee, Chiral anomalies, higher dimensions and differential geometry, Nucl. Phys. B 239 (1984) 477 [inSPIRE].

[15] H. Leutwyler, Chiral fermion determinants and their anomalies, Phys. Lett. B 152 (1985) 78 [INSPIRE].

[16] W.D. Linch, III and G. Tartaglino-Mazzucchelli, Six-dimensional supergravity and projective superfields, JHEP 08 (2012) 075 [arXiv: 1204.4195] [INSPIRE].

[17] D. Butter, S.M. Kuzenko and J. Novak, The linear multiplet and ectoplasm, JHEP 09 (2012) 131 [arXiv: 1205.6981] [INSPIRE].

[18] L. Mezincescu, On the superfield formulation of O(2) supersymmetry, JINR-P2-12572 (1979). 
[19] I.A. Batalin and G.A. Vilkovisky, Quantization of gauge theories with linearly dependent generators, Phys. Rev. D 28 (1983) 2567 [Erratum ibid. D 30 (1984) 508] [INSPIRE].

[20] J. Wess and B. Zumino, Consequences of anomalous Ward identities, Phys. Lett. B 37 (1971) 95 [INSPIRE].

[21] P. Binetruy, G. Girardi and R. Grimm, Supergravity couplings: a geometric formulation, Phys. Rept. 343 (2001) 255 [hep-th/0005225] [INSPIRE].

[22] A.S. Galperin, E.A. Ivanov, V.I. Ogievetsky and E.S. Sokatchev, Harmonic superspace, Cambridge University Press, Cambridge U.K. (2001).

[23] I.L. Buchbinder, S.M. Kuzenko and B.A. Ovrut, On the $D=4, N=2$ nonrenormalization theorem, Phys. Lett. B 433 (1998) 335 [hep-th/9710142] [INSPIRE].

[24] E.A. Ivanov, A.V. Smilga and B.M. Zupnik, Renormalizable supersymmetric gauge theory in six dimensions, Nucl. Phys. B 726 (2005) 131 [hep-th/0505082] [INSPIRE].

[25] I.L. Buchbinder and N.G. Pletnev, Construction of $6 D$ supersymmetric field models in $N=(1,0)$ harmonic superspace, Nucl. Phys. B 892 (2015) 21 [arXiv:1411.1848] [INSPIRE].

[26] I.L. Buchbinder and N.G. Pletnev, Leading low-energy effective action in the $6 D$ hypermultiplet theory on a vector/tensor background, Phys. Lett. B 744 (2015) 125 [arXiv: 1502.03257] [INSPIRE].

[27] A. Galperin, E. Ivanov, S. Kalitsyn, V. Ogievetsky and E. Sokatchev, Unconstrained $N=2$ Matter, Yang-Mills and supergravity theories in harmonic superspace, Class. Quant. Grav. 1 (1984) 469 [Erratum ibid. 2 (1985) 127] [INSPIRE].

[28] U. Lindström and M. Roček, $N=2$ super Yang-Mills theory in projective superspace, Commun. Math. Phys. 128 (1990) 191 [InSPIRE].

[29] D. Butter and S.M. Kuzenko, New higher-derivative couplings in $4 D N=2$ supergravity, JHEP 03 (2011) 047 [arXiv: 1012.5153] [INSPIRE].

[30] S.M. Kuzenko, Projective superspace as a double punctured harmonic superspace, Int. J. Mod. Phys. A 14 (1999) 1737 [hep-th/9806147] [INSPIRE].

[31] P.S. Howe, K.S. Stelle and P.C. West, $N=1 D=6$ harmonic superspace, Class. Quant. Grav. 2 (1985) 815 [INSPIRE].

[32] B.M. Zupnik, Six-dimensional supergauge theories in the harmonic superspace, Sov. J. Nucl. Phys. 44 (1986) 512 [Yad. Fiz. 44 (1986) 794] [INSPIRE].

[33] B.M. Zupnik, The action of the supersymmetric $N=2$ gauge theory in harmonic superspace, Phys. Lett. B 183 (1987) 175 [inSPIRE].

[34] U. Lindström and M. Roček, New HyperKähler metrics and new supermultiplets, Commun. Math. Phys. 115 (1988) 21 [INSPIRE].

[35] S.M. Kuzenko, Lectures on nonlinear $\sigma$-models in projective superspace, J. Phys. A 43 (2010) 443001 [arXiv: 1004.0880] [inSPIRE].

[36] S.M. Kuzenko and G. Tartaglino-Mazzucchelli, $N=4$ supersymmetric Yang-Mills theories in $A d S_{3}, J H E P 05$ (2014) 018 [arXiv: 1402.3961] [INSPIRE].

[37] D. Butter, S.M. Kuzenko, J. Novak and G. Tartaglino-Mazzucchelli, Conformal supergravity in five dimensions: new approach and applications, JHEP 02 (2015) 111 [arXiv:1410.8682] [INSPIRE].

[38] A. Galperin, E.A. Ivanov, V. Ogievetsky and E. Sokatchev, Harmonic supergraphs. Green functions, Class. Quant. Grav. 2 (1985) 601 [INSPIRE]. 